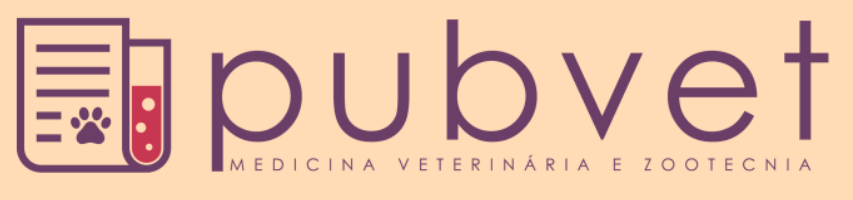

https://doi.org/10.22256/pubvet.v12n2a34.1-9

\title{
Desempenho, qualidade da carcaça e carne de ovinos de descarte de diferentes idades e gêneros
}

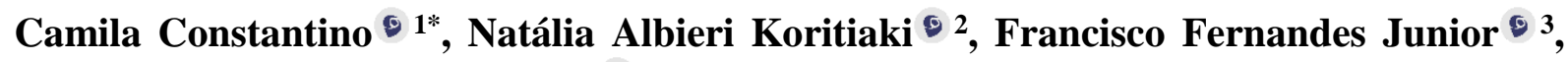 \\ Edson Luis de Azambuja Ribeiro ${ }^{0} 4$
}

${ }^{1}$ Universidade Estadual de Londrina, Zootecnia, Londrina, Paraná, Brasil. ${ }^{2}$ Centro Universitário Filadélfia, Medicina Veterinária, Londrina, Paraná, Brasil. nataliaalbieri@hotmail.com; ${ }^{3}$ Universidade Estadual de Londrina, Zootecnia, Londrina, Paraná, Brasil. ffjunior_zoo@ hotmail.com; ${ }^{4}$ Universidade Estadual de Londrina, Zootecnia, Londrina, Paraná, Brasil.elar@uel.br

*Autor para correspondência, E-mail: caconstantino@hotmail.com

\begin{abstract}
RESUMO. As carcaças de animais de descarte ou mais velhos apresentam, geralmente, excesso de gordura, a cor da carne mais escura, carne mais firme e apresentam sabor e aroma mais acentuados. Objetivou-se com este trabalho mensurar o desempenho, qualidade da carcaça e carne de ovinos de diferentes categorias. Foram utilizados 25 animais, sendo 5 borregas, 5 cordeiros, 5 machos inteiros, 5 machos castrados e 5 ovelhas. Após um período de 50 dias de pastejo diário e suplementação no período da noite os animais foram abatidos. Foram realizadas mensurações na carcaça e carne dos animais. Os dados foram submetidos a análise de variância e teste Tukey a 5\%. Em relação ao desempenho, biometria e avaliações de carcaça, os machos castrados, inteiros e ovelhas apresentaram os maiores valores, porém é interessante ressaltar que os cordeiros e borregas apresentaram resultados satisfatórios. Outro ponto importante é que o rendimento verdadeiro, a força de cisalhamento e a aceitabilidade global da amostra na análise sensorial foram semelhantes entre todas as categorias. Em relação ao tamanho de cortes o que deve ser levado em conta é o que o mercado consumidor almeja, consumir cortes grandes ou pequenos. Quanto a qualidade da carne, animais adultos apresentaram mais gordura, o que pode ser interessante para garantir uma melhor carcaça e qualidade sensorial da carne, desde que não seja um excesso que aumente as perdas no toalet da carcaça ou piore o odor da carne.
\end{abstract}

Palavras chave: Borrega, cordeiro, macho castrado, macho não castrado, ovelha

\section{Performance, carcass quality and meat of sheep from different ages and genres}

ABSTRACT. Several authors commented that the inadequate quality of sheep meat is due to the low quality of the meat of discarded animals, like adult animals. The carcasses are excess fat; the meat color is darker, meat firmer, and has a more pronounced taste and aroma. The objective of this work was to measure the performance, carcass quality and meat of sheep of different categories. Twenty five animals, 5 lambs, 5 lambs, 5 whole males, 5 castrated males and 5 sheep were used. After a period of 50 days of daily grazing and supplementation at night the animals were slaughtered. Measurements were made on the carcass and meat of the animals. Data were submitted to analysis of variance and Tukey test at 5\%. Regarding performance, biometrics and carcass evaluations, castrated males, whole males and sheep presented the highest values, but it is interesting to note that lambs and lambs presented satisfactory results. Another important point is that the true yield, shear force and overall acceptability of the sample in the sensory analysis were similar across all categories. Regarding the size of cuts what should be taken into account is what the consumer market expects, consuming large or small cuts. As for the quality of the meat, 
adult animals presented more fat, which may be interesting to guarantee a better carcass and sensorial quality of the meat, as long as it is not an excess that increases the losses in the carcass toilet or worse the odor of the meat.

Keywords: lamb, castrated male, entire male, ewe, lamb

\section{Rendimiento, calidad de la canal y carne de ovino de descarte de diferentes edades y géneros}

RESUMEN. Las canales de animales de descarte o más viejos presentan, generalmente, exceso de grasa, el color de la carne más oscura, carne más firme y presentan sabor y aroma más acentuados. Se objetivó con este trabajo medir el desempeño, calidad de la canal y carne de ovinos de diferentes categorías. Se utilizaron 25 animales, siendo 5 borregas, 5 corderos, 5 machos enteros, 5 machos castrados y 5 ovejas. Después de un período de 50 días de pastoreo diario y suplementación en el período de la noche los animales fueron sacrificados. Se realizaron mediciones en la canal y carne de los animales. Los datos fueron sometidos a análisis de varianza y test Tukey al 5\%. En cuanto al rendimiento, la biometría y las evaluaciones de la canal, los machos castrados, enteros y ovejas presentaron los mayores valores, pero es interesante resaltar que los corderos y las borregas presentaron resultados satisfactorios. Otro punto importante es que el rendimiento real, la fuerza de cizallamiento y la aceptación global de la muestra en el análisis sensorial fueron similares entre todas las categorías. En cuanto al tamaño de cortes lo que debe tenerse en cuenta es lo que anhela el mercado consumidor, consumir cortes grandes o pequeños. En cuanto a la calidad de la carne, los animales adultos presentaron más grasa, lo que puede ser interesante para garantizar una mejor canal y calidad sensorial de la carne, siempre que no sea un exceso que aumente las pérdidas en el toalet de la canal o inferior el olor de la carne

Palabras chave: Borregas, cordero, macho castrado, macho entero, oveja

\section{Introdução}

Segundo o ANUALPEC (2017) o consumo per capita foi $37,4 \mathrm{~kg}$ para carne bovina, $43,9 \mathrm{~kg}$ para carne de aves e 14,1 kg para carne suína. Em uma entrevista, Juan Ferelli Souza - Embrapa Caprinos e Ovinos - comenta que no ano de 2012 foi consumido no Brasil aproximadamente 89 mil toneladas de carne ovina (Beraldo, 2014), levando em conta que no mesmo ano a população foi de 198,7 milhões de pessoas. Assim, podemos estimar um consumo de $0,45 \mathrm{~kg} / \mathrm{pessoa} /$ ano. O baixo consumo de carne ovina no Brasil se deve à falta de hábito do consumidor, irregularidade da oferta, qualidade inadequada do produto colocado à venda e à má apresentação do produto oferecido no mercado interno (SEBRAE, 2005).

Vários autores comentam que a qualidade inadequada da carne ovina se deve a baixa qualidade da carne de animais de descarte, como animais adultos. A idade tem sido relacionada com o aumento do flavour, ou o aumento da intensidade. Portanto, carcaças de animais mais velhos, como ovelhas de descarte, podem apresentar vários problemas de qualidade. As carcaças apresentam excesso de gordura, a cor da carne é mais escura, carne mais firme e apresentam sabor e aroma mais acentuados (Beserra et al., 1999). O que geralmente ocorre com o aumento da idade é o aumento da adiposidade da carcaça e isso sim pode afetar o odor da carne por aumentar a deposição de ácidos graxos de cadeia ramificada. Com o avanço da idade ou peso, a carne proveniente de machos não castrados pode apresentar uma redução da maciez e de outras características qualitativas da carne. A idade limite para o abate de machos não castrados, sem que a qualidade da carne seja negativamente afetada, depende de vários fatores, tais como peso, sistema de criação e alimentação (Beermann et al., 1995).

Objetivou-se com este trabalho avaliar características de desempenho, biometria, qualidade da carcaça e carne de ovinos de diferentes gêneros e idades, visando evidenciar reais diferenças que possam denegrir a imagem do produto.

\section{Material e Métodos \\ O experimento foi realizado na Fazenda Escola da Universidade Estadual de Londrina (UEL),}


onde foram utilizados 25 animais, sendo: 5 borregas (12 meses de idade), 5 cordeiros ( 6 meses de idade), 5 machos não castrados (24 meses de idade), 5 machos castrados ( 24 meses de idade) e 5 ovelhas ( 96 meses de idade). Estes animais eram oriundos do rebanho ovinos na UEL.

O experimento foi realizado no período de terminação destes animais e o delineamento experimental foi completamente casualizado, sendo os animais divididos em categorias (borrega, cordeiro, macho não castrado, macho castrado e ovelha) totalizando 5 animais por tratamento. Os animais passavam o período do dia em pasto de coast cross e a noite ficavam alojados no curral, onde recebiam suplementação ( $2 \%$ do peso vivo) à base de silagem de sorgo e concentrado com milho e soja. O período experimental foi de 50 dias.

Semanalmente foi realizada a pesagem dos animais. Antes do abate os animais foram pesados, mensurado o escore de condição corporal e realizadas as medidas biométricas in vivo com o auxílio de uma fita métrica e uma régua de madeira com animal mantido em posição correta de aprumos. Foi determinado: comprimento corporal, comprimento da perna, altura do anterior, altura do posterior, perímetro torácico, perímetro da perna, largura da garupa e largura do peito de cada animal, segundo a metodologia de Osório \& Osório (2005).

Após jejum sólido de 16 horas, os animais foram transportados por $40 \mathrm{~km}$ ao frigorífico onde permaneceram em baia de espera. $\mathrm{O}$ abate foi realizado conforme as normas de abate humanitário, em frigorífico comercial com inspeção estadual.

Foi coletado o trato gastrointestinal, que foi pesado cheio e vazio para obtenção do peso corporal vazio (Osório \& Osório, 2005). As carcaças foram pesadas logo após o abate (peso da carcaça quente) e após 24 horas de resfriamento a $2^{\circ} \mathrm{C}$ (peso da carcaça fria). Os rendimentos de carcaça foram calculados pelas porcentagens dos pesos da carcaça quente e fria em relação ao peso vivo. Também foi realizado o rendimento verdadeiro, que é o peso da carcaça quente em relação ao peso corporal vazio (Osório \& Osório, $\underline{2005}$ ).

Foi realizada avaliação de acabamento (1 gordura de cobertura ausente a 4 - gordura de cobertura abundante) e conformação (1 - côncavo a 5 - convexo) utilizando padrões fotográficos
(Cañeque \& Sañudo, 2000). Foram realizadas medidas de comprimento de carcaça e profundidade torácica, comprimento, perímetro e profundidade de perna e braço (Osório \& Osório, 2005).

As meias carcaças esquerdas foram seccionadas entre a $12^{\mathrm{a}}$ e $13^{\mathrm{a}}$ costelas para avaliação da profundidade e largura do músculo, área de olho de lombo e espessura de gordura subcutânea do músculo longissimus dorsi (Osório \& Osório, 2005, Cezar \& Sousa, 2007). A paleta esquerda foi levada ao laboratório para determinação da composição tecidual.

No laboratório, o Longissimus dorsi foi dividido em amostras de 2 a $4 \mathrm{~cm}$ de espessura para análises de perda de água por pressão, $\mathrm{pH}$, marmoreio, cor, perda de água no descongelamento e cocção, força de cisalhamento, composição centesimal e avaliação sensorial.

$\mathrm{O}$ grau de marmoreio foi avaliado subjetivamente utilizando padrões fotográficos (1 - traços de marmoreio e 10 - marmoreio abundante) (AMSA, 2001). A cor foi avaliada após 30 minutos do corte para oxigenação da mioglobina, utilizando o colorímetro portátil Color reader CR-10 Konica Minolta ${ }^{\circledR}$ (Konica Minolta, Chiyoda, TKO) (CIE, 1986) para avaliação dos componentes L* (luminosidade), $\mathrm{a}^{*}$ (componente vermelho-verde) e $\mathrm{b}^{*}$ (componente amarelo-azul) que foram expressos no sistema de cor CIELAB*. Com esses valores, fez-se o cálculo do ângulo de tonalidade ( $\mathrm{h}^{*}$ ) pela equação $\mathrm{h}^{*}=$ $\tan -1\left(b^{*} / a^{*}\right)$, e o índice de saturação, ou croma, $\left(c^{*}\right)$ a partir da equação $\mathrm{c}^{*}=\left(\mathrm{a}^{* 2}+\mathrm{b}^{* 2}\right) 0,5$.

Foi realizada medida do $\mathrm{pH}$ final, utilizando um aparelho portátil HI99163 HANNA ${ }^{\circledR}$ (Hanna Instruments, Tamboré, SP) com eletrodo de inserção. As amostras restantes foram embaladas individualmente e congeladas a $-18^{\circ} \mathrm{C}$ para análises posteriores.

O preparo das amostras para a avaliação da força de cisalhamento foi realizado em forno elétrico pré-aquecido a $180^{\circ} \mathrm{C}$. Os bifes de $3 \mathrm{~cm}$ de altura foram assados até atingirem $40{ }^{\circ} \mathrm{C}$ internamente, então foram virados e assados até atingirem $71^{\circ} \mathrm{C}$ internamente (AMSA, 1995). Durante este processo foi avaliada a perda de água no descongelamento e cocção por meio de pesagens da amostra congelada, descongelada e cozida.

Para a obtenção das amostras foi utilizado um amostrador cilíndrico. Foram utilizados três bifes 
por animal, de cada bife foram retiradas duas subamostras de aproximadamente $1,25 \mathrm{~cm}$ de espessura (Wheeler et al., 1997, Ramos \& Gomide, 2007) e cada subamostra foi cisalhada uma única vez, totalizando seis leituras por animal. A força de cisalhamento foi objetivamente medida por meio da utilização de um texturômetro CT3 Texture Analyser Brookfield ${ }^{\circledR}$ (Brookfield engineering, Middleboro, MA) com lâmina warner-bratzler de $3 \mathrm{~mm}$ de espessura.

A análise centesimal, que quantifica umidade, lipídios, proteína e cinzas, foi realizada conforme a metodologia descrita por ( $\underline{\text { AOAC, 2005) }}$.

A análise sensorial foi realizada por um grupo de 12 provadores treinados utilizando uma escala de intensidade e caracterização (ABNT, 1998), sendo avaliadas a intensidade e caracterização do odor, maciez, suculência e aceitabilidade global. Cada provador recebeu a ficha de avaliação sensorial, copo de água para limpeza da boca, bolacha de água e sal para rinsagem da boca e recipiente com café para limpeza do olfato. As amostras foram preparadas da mesma forma que para força de cisalhamento.

Os dados foram submetidos à análise de variância e teste Tukey a $5 \%$ de probabilidade, utilizando o pacote estatístico do SAS (2004), tendo como variáveis independentes as categorias animais (cordeiro, macho castrado, ovelha, borrega, macho não castrado e ovelha).

\section{Resultados e Discussão}

Ao avaliar diferentes categorias animais era de se esperar diferenças em peso e tamanho (Tabela 1). O peso inicial foi semelhante entre os animais adultos, ovelha, macho não castrado e macho castrado; e semelhante entre os cordeiros e as borregas. Neste experimento foram utilizados animais de descarte, ovelhas no fim da sua vida produtiva, machos e borregas que por vários motivos não foram aptos a reprodução e cordeiros de mamadeira.

Tabela 1. Médias observadas para o desempenho e biometria de ovinos de diferentes categorias animais.

\begin{tabular}{|c|c|c|c|c|c|c|c|c|}
\hline \multirow{2}{*}{ Variáveis } & \multicolumn{5}{|c|}{ Categorias } & \multirow{2}{*}{$\begin{array}{l}\mathrm{CV} \\
(\%)\end{array}$} & \multirow{2}{*}{$\begin{array}{l}\text { Média } \\
\text { geral }\end{array}$} & \multirow{2}{*}{$P$} \\
\hline & Borrega & Cordeiro & Macho castrado & oMacho não castrado & Ovelha & & & \\
\hline Peso inicial (kg) & $19,00 \mathrm{~b}$ & $12,00 \mathrm{~b}$ & $41,70 \mathrm{a}$ & $38,50 \mathrm{a}$ & $38,00 \mathrm{a}$ & 21,98 & 29,83 & $<0,0001$ \\
\hline Peso final (kg) & $22,80 \mathrm{~b}$ & $14,10 \mathrm{~b}$ & $46,20 \mathrm{a}$ & $44,60 \mathrm{a}$ & $45,50 \mathrm{a}$ & 20,38 & 34,64 & $<0,0001$ \\
\hline Ganho médio diário (g) & $0,112 \mathrm{bc}$ & $0,046 \mathrm{c}$ & $0,134 \mathrm{~b}$ & $0,178 \mathrm{ab}$ & $0,218 \mathrm{a}$ & 30,27 & 0,14 & $<0,0001$ \\
\hline Escore & 2,75 & 2,6 & 3,4 & 2,85 & 2,55 & 17,93 & 2,83 & 0,1002 \\
\hline Altura de dorso $(\mathrm{cm})$ & $60,80 \mathrm{~b}$ & $56,40 \mathrm{~b}$ & 71,20 a & $72,00 \mathrm{a}$ & $72,40 \mathrm{a}$ & 8,03 & 66,56 & 0,0002 \\
\hline Altura de posterior $(\mathrm{cm})$ & $60,40 \mathrm{~b}$ & $54,60 \mathrm{~b}$ & $72,00 \mathrm{a}$ & $70,60 \mathrm{a}$ & $71,40 \mathrm{a}$ & 6,33 & 65,8 & $<0,0001$ \\
\hline Perímetro torácico $(\mathrm{cm})$ & $65,00 \mathrm{~b}$ & $58,20 \mathrm{~b}$ & $87,00 \mathrm{a}$ & $80,40 \mathrm{a}$ & $88,80 \mathrm{a}$ & 7,97 & 75,88 & $<0,0001$ \\
\hline Comprimento corporal $(\mathrm{cm})$ & $50,80 \mathrm{~b}$ & $42,60 \mathrm{~b}$ & $62,80 \mathrm{a}$ & $62,20 \mathrm{a}$ & 64,40 a & 8,24 & 56,56 & $<0,0001$ \\
\hline Comprimento de perna $(\mathrm{cm})$ & $25,80 \mathrm{~b}$ & $24,00 \mathrm{~b}$ & $33,20 \mathrm{a}$ & $33,40 \mathrm{a}$ & $30,60 \mathrm{a}$ & 8,6 & 29,4 & $<0,0001$ \\
\hline Perímetro de perna $(\mathrm{cm})$ & $29,00 \mathrm{c}$ & $22,60 \mathrm{~d}$ & 37,80 a & $36,00 \mathrm{ab}$ & $32,40 \mathrm{bc}$ & 7,93 & 31,56 & $<0,0001$ \\
\hline Largura de peito $(\mathrm{cm})$ & $15,80 \mathrm{~b}$ & $13,60 \mathrm{~b}$ & $21,20 \mathrm{a}$ & $20,80 \mathrm{a}$ & $20,20 \mathrm{a}$ & 9,95 & 18,32 & $<0,0001$ \\
\hline Largura de garupa $(\mathrm{cm})$ & $18,60 \mathrm{~b}$ & $14,80 \mathrm{c}$ & $22,60 \mathrm{a}$ & $21,60 \mathrm{ab}$ & $24,2 \mathrm{a}$ & 9,14 & 20,36 & $<0,0001$ \\
\hline
\end{tabular}

$\overline{\mathrm{CV}}$ - Coeficiente de variação; $\mathrm{P}$ - Probabilidade; $\mathrm{a}, \mathrm{b}, \mathrm{c}, \mathrm{d}, \mathrm{e}-$ Médias na linha seguidas por letras diferem $(\mathrm{P}<0,05)$ entre si.

Após um período de avaliação de 50 dias, mesmo com todos os animais ganhando em média $5 \mathrm{~kg}$ de peso corporal, essas diferenças se mantiveram. Os animais adultos tiveram uma deposição de tecido adiposo e os animais jovens tiveram seu crescimento acelerado, apresentando ganho de peso devido ao crescimento ósseo e muscular.

Os animais apresentaram (Tabela 1) ganho médio diário de 46 gramas/dia a 218 gramas/dia, para cordeiros e ovelhas, respectivamente. Em relação ao escore corporal, não houve diferença (P
$>0,05$ ). Na biometria (Tabela 1) dos animais, podemos observar que todos os parâmetros avaliados apresentaram comportamento semelhante, ovelhas, machos não castrados e castrados apresentam os maiores valores, seguidos pelas borregas e pelos cordeiros. Isto se deve a diferença de idade e o fato de que as borregas e os cordeiros ainda se encontravam em fase de crescimento.

No peso de carcaça quente, fria e peso corporal vazio, assim como nos rendimentos de carcaça quente e fria (Tabela 2), observou-se o mesmo 
comportamento das outras variáveis até o momento. Maiores pesos para as ovelhas, machos inteiros e castrados e menores para as borregas e cordeiros. Entretanto quando se observa o rendimento verdadeiro, não há diferença entre as categorias, ou seja, quando se ignora o peso do conteúdo gastrointestinal - que é utilizado o peso corporal vazio para o cálculo deste rendimento, esta diferença não ocorre.

Atualmente há uma busca por maior rendimento das carcaças, entretanto nem sempre as carcaças com maiores rendimentos são melhores, pois podem apresentar maiores depósitos de tecido adiposo. Deve-se buscar a produção de animais com alto rendimento de carcaça, desde que ele seja composto por alta proporção de músculos e quantidade adequada de gordura, visando proteger a carcaça durante o resfriamento e conferir atributos sensoriais desejáveis pelo consumidor (Pinheiro et al., 2009).

A perda na refrigeração é altamente correlacionada com o acabamento da carcaça, isto por que o acabamento atua como isolante térmico para a carcaça, reduzindo a velocidade da queda da temperatura. Este processo evita o encurtamento pelo frio e reduz as perdas de água além de garantir que a superfície da carcaça não resseque (Lawrie, 1981, Pearce et al., 2011). Desta forma, as maiores perdas foram observadas para as carcaças com menores acabamentos de gordura, dos cordeiros, seguidas pelas borregas e machos inteiros. Os machos castrados e as ovelhas apresentam as menores perdas. Todos os valores observados neste experimento estavam abaixo dos valores aceitáveis para a carcaça ovina 3 a $4 \%$ (Siqueira \& Fernandes, 1999).

Tabela 2. Médias observadas para características de carcaça de ovinos de diferentes categorias animais.

\begin{tabular}{|c|c|c|c|c|c|c|c|c|}
\hline \multirow{2}{*}{ Variáveis } & \multicolumn{5}{|c|}{ Categorias } & \multirow{2}{*}{$\begin{array}{l}\mathrm{CV} \\
(\%)\end{array}$} & \multirow{2}{*}{$\begin{array}{c}\text { Média } \\
\text { geral }\end{array}$} & \multirow{2}{*}{$\mathrm{P}$} \\
\hline & Borrega & Cordeiro & Macho castrado & loMacho não castrado & Ovelha & & & \\
\hline Peso de carcaça quente $(\mathrm{kg})$ & $8,90 \mathrm{~b}$ & $5,15 \mathrm{~b}$ & $20,95 \mathrm{a}$ & $19,20 \mathrm{a}$ & $18,21 \mathrm{a}$ & 22,21 & 14,48 & $<0,0001$ \\
\hline Peso de carcaça fria $(\mathrm{kg})$ & $8,63 \mathrm{~b}$ & $4,96 \mathrm{~b}$ & $20,45 \mathrm{a}$ & $18,71 \mathrm{a}$ & $17,72 \mathrm{a}$ & 22,48 & 14,09 & $<0,0001$ \\
\hline Peso corporal vazio $(\mathrm{kg})$ & $18,23 \mathrm{~b}$ & $11,04 \mathrm{~b}$ & $41,09 \mathrm{a}$ & 37,79 a & $37,42 \mathrm{a}$ & 20,39 & 29,11 & $<0,0001$ \\
\hline Rendimento carcaça quente $(\%)$ & $39,07 \mathrm{~cd}$ & $36,52 \mathrm{~d}$ & 45,29 a & $42,89 \mathrm{ab}$ & $40,00 \mathrm{bc}$ & 4,36 & 40,75 & $<0,0001$ \\
\hline Rendimento carcaça fria (\%) & $37,87 \mathrm{~cd}$ & $35,18 \mathrm{~d}$ & $44,20 \mathrm{a}$ & $41,76 \mathrm{ab}$ & $38,92 \mathrm{bc}$ & 4,32 & 39,59 & $<0,0001$ \\
\hline Rendimento verdadeiro (\%) & 48,92 & 46,71 & 50,99 & 50,42 & 48,69 & 4,75 & 49,15 & 0,0697 \\
\hline Perda na refrigeração (\%) & $3,06 \mathrm{ab}$ & $3,66 \mathrm{a}$ & $2,40 \mathrm{~b}$ & $2,66 \mathrm{ab}$ & $2,68 \mathrm{ab}$ & 19,57 & 2,9 & 0,0204 \\
\hline
\end{tabular}

$\overline{\mathrm{CV}}$ - Coeficiente de variação; $\mathrm{P}$ - Probabilidade; $\mathrm{a}, \mathrm{b}, \mathrm{c}, \mathrm{d}, \mathrm{e}-$ Médias na linha seguidas por letras diferem $(\mathrm{P}<0,05)$ entre si.

Machos castrados e ovelhas apresentaram melhor conformação seguida pelos machos inteiros e depois os cordeiros e borregas (Tabela 3). Importante ressaltar que as conformações observadas foram ruins e regulares. Nenhuma carcaça de nenhuma categoria apresentou uma boa conformação. O mesmo observou-se para o acabamento, que foi insuficiente nas borregas e cordeiros, reiterando que eram animais em fase de crescimento e com tamanho insatisfatório para que apresentassem carcaças com características desejáveis ao mercado consumidor.

Quanto às medidas da carcaça (Tabela 3), as maiores carcaças apresentaram as maiores medidas, ou seja, os machos castrados e inteiros, seguidos pelas ovelhas, borregas e por último os cordeiros. O perímetro de perna é indicativo de conformação e musculosidade (Yáñez et al., 2004). O índice de compacidade corporal relaciona $o$ peso de carcaça fria com $o$ comprimento de carcaça, e é utilizado para indicar a proporção de parte comestível, sem levar em conta a proporção de músculo e gordura (Cezar \& Sousa, 2007). Neste experimento o maior índice foi dos machos castrados, inteiros e ovelhas, seguidos pelas borregas e cordeiros. As medidas do Longissimus dorsi também são altamente correlacionadas com o grau de musculosidade da carcaça e apresentou o mesmo comportamento que o restante das variáveis.

Quanto ao peso dos cortes (Tabela 4), os maiores pesos foram para as categorias das ovelhas, machos inteiros e castrados e inferiores para as borregas e cordeiros, o que era de se esperar já que eram animais que estavam em fase de crescimento. Quanto à proporção dos cortes, apesar de algumas diferenças significativas, as proporções encontradas foram muito próximas, isto se deve a lei da harmonia anatômica (Lawrie, 1981).

Em relação à composição tecidual da paleta (Tabela 4), não houve diferença $(P>0,05)$ para a quantidade de músculo e osso, porém a quantidade 
de gordura foi afetada. Os maiores valores foram observados para os machos castrados, seguido pelas ovelhas, machos inteiros, cordeiros e por último as borregas com menos de $8 \%$ de gordura. Segundo Gonçalves et al. (2004), a carcaça e a carne de animais machos não castrados são reconhecidas por apresentar menor quantidade de gordura do que machos castrados e estas menos do que fêmeas, e esta variação deve-se basicamente aos diferentes níveis de hormônios sexuais.

Tabela 3. Médias observadas para características de carcaça de ovinos de diferentes categorias animais.

\begin{tabular}{|c|c|c|c|c|c|c|c|c|}
\hline \multirow{2}{*}{ Variáveis } & \multicolumn{5}{|c|}{ Categorias } & \multirow{2}{*}{\multicolumn{2}{|c|}{$\begin{array}{l}\text { CV Média } \\
(\%) \text { geral }\end{array}$}} & \multirow{2}{*}{$\mathrm{P}$} \\
\hline & \multicolumn{4}{|c|}{ Borrega CordeiroMacho castradoMacho não castrado } & \multirow{2}{*}{$\frac{\text { Ovelha }}{2,40 \mathrm{a}}$} & & & \\
\hline$\overline{\text { Conformação }}$ & $1,00 \mathrm{~b}$ & $1,00 \mathrm{~b}$ & $2,60 \mathrm{a}$ & $1,80 \mathrm{ab}$ & & 38,54 & 41,76 & $\overline{0,0021}$ \\
\hline Acabamento & $1,20 \mathrm{c}$ & $1,00 \mathrm{c}$ & $3,60 \mathrm{a}$ & $2,40 \mathrm{~b}$ & $3,00 \mathrm{ab}$ & 26,79 & 2,24 & $<0,0001$ \\
\hline Comprimento de carcaça $(\mathrm{cm})$ & $54,60 \mathrm{bc}$ & $46,00 \mathrm{c}$ & 66,80 a & 65,60 a & $63,80 \mathrm{ab}$ & 8,41 & 59,36 & $<0,0001$ \\
\hline Profundidade torácica $(\mathrm{cm})$ & $23,00 \mathrm{~b}$ & $19,80 \mathrm{~b}$ & $29,60 \mathrm{a}$ & $28,20 \mathrm{a}$ & $30,40 \mathrm{a}$ & 6,93 & 26,2 & $<0,0001$ \\
\hline Índice de compacidade corporal & $0,156 \mathrm{~b}$ & $0,108 b$ & $0,306 \mathrm{a}$ & $0,282 \mathrm{a}$ & 0,278 a & 17,89 & 0,22 & $<0,0001$ \\
\hline Comprimento de perna $(\mathrm{cm})$ & $37,50 \mathrm{~b}$ & $34,80 \mathrm{~b}$ & $43,10 \mathrm{a}$ & $43,60 \mathrm{a}$ & $42,20 \mathrm{a}$ & 5,32 & 40,24 & $<0,0001$ \\
\hline Perímetro de perna $(\mathrm{cm})$ & $31,30 \mathrm{~b}$ & $26,06 \mathrm{~b}$ & $41,52 \mathrm{a}$ & $40,00 \mathrm{a}$ & $40,50 \mathrm{a}$ & 7,77 & 35,98 & $<0,0001$ \\
\hline Profundidade de perna $(\mathrm{cm})$ & $6,80 \mathrm{bc}$ & $4,80 \mathrm{c}$ & $10,10 \mathrm{a}$ & $10,00 \mathrm{a}$ & $8,80 a b$ & 18,33 & 38,1 & $<0,0001$ \\
\hline Comprimento de braço $(\mathrm{cm})$ & 17,1 & 17,4 & 19,6 & 19,3 & 19,7 & 8,92 & 18,62 & 0,0485 \\
\hline Perímetro de braço $(\mathrm{cm})$ & $13,80 \mathrm{~b}$ & $11,40 \mathrm{c}$ & $17,70 \mathrm{a}$ & 17,90 a & $17,00 \mathrm{a}$ & 7,94 & 15,56 & $<0,0001$ \\
\hline Profundidade de braço $(\mathrm{cm})$ & $4,10 \mathrm{~b}$ & $2,20 \mathrm{c}$ & 5,80 a & $6,00 \mathrm{a}$ & $4,90 \mathrm{ab}$ & 18,83 & 33,6 & $<0,0001$ \\
\hline Espessura de gordura do músculo (mm) & $0,70 \mathrm{~b}$ & $0,75 \mathrm{~b}$ & $2,37 \mathrm{a}$ & $1,16 \mathrm{ab}$ & $1,83 \mathrm{ab}$ & 49,05 & 51,36 & 0,0026 \\
\hline Profundidade do músculo (mm) & $44,95 \mathrm{bc}$ & $38,39 \mathrm{c}$ & 55,66 a & $57,54 \mathrm{a}$ & $50,68 \mathrm{ab}$ & 7,93 & 49,44 & $<0,0001$ \\
\hline Largura do músculo (mm) & $20,87 \mathrm{bc}$ & $16,20 \mathrm{c}$ & 30,16 a & $28,41 \mathrm{ab}$ & $26,53 \mathrm{ab}$ & 16,32 & 24,43 & $<0,0001$ \\
\hline Área de olho de lombo $\left(\mathrm{cm}^{2}\right)$ & $10,15 b$ & $5,85 \mathrm{c}$ & $13,85 \mathrm{ab}$ & $14,80 \mathrm{a}$ & $11,45 \mathrm{ab}$ & 18,53 & 311,22 & $<0,0001$ \\
\hline
\end{tabular}

CV - Coeficiente de variação; $\mathrm{P}$ - Probabilidade; a, b, c, d, e - Médias na linha seguidas por letras diferem $(\mathrm{P}<0,05)$ entre si.

Sobre as características da carne ovina de animais de diferentes categorias (Tabela 5), não houve diferença $(\mathrm{P}>0,05)$ no $\mathrm{pH}$ da carne, e os valores observados estavam dentro dos limites considerados normais para a espécie, de 5,5 a 5,8, segundo Zeola et al. (2007), mostrando que ovinos dificilmente apresentam problemas relacionados ao $\mathrm{pH}$ da carne, como carne DFD (escura, firme e seca).

Tabela 4. Médias observadas para peso e proporção de cortes e composição tecidual de ovinos de diferentes categorias animais.

\begin{tabular}{|c|c|c|c|c|c|c|c|c|}
\hline \multirow[b]{2}{*}{ Variáveis } & \multicolumn{5}{|c|}{ Categorias } & \multirow{2}{*}{$\begin{array}{l}\mathrm{CV} \\
(\%)\end{array}$} & \multirow{2}{*}{$\begin{array}{l}\text { Média } \\
\text { geral }\end{array}$} & \multirow[b]{2}{*}{$\mathrm{P}$} \\
\hline & Borrega & Cordeiro & $\begin{array}{l}\text { Macho } \\
\text { castrado }\end{array}$ & $\begin{array}{l}\text { Macho não } \\
\text { castrado }\end{array}$ & Ovelha & & & \\
\hline Pescoço (kg) & $0,52 \mathrm{~b}$ & $0,39 \mathrm{~b}$ & $1,38 \mathrm{a}$ & $1,32 \mathrm{a}$ & $1,21 \mathrm{a}$ & 32,87 & 0,98 & $<0,0001$ \\
\hline Pescoço (\%) & $11,84 \mathrm{~b}$ & $15,85 \mathrm{a}$ & $12,99 \mathrm{ab}$ & $14,44 \mathrm{ab}$ & $13,29 \mathrm{ab}$ & 12,05 & 13,68 & 0,0117 \\
\hline Pernil (kg) & $1,51 \mathrm{~b}$ & $0,85 \mathrm{~b}$ & $3,10 \mathrm{a}$ & $2,78 \mathrm{a}$ & $2,77 \mathrm{a}$ & 19,43 & 2,2 & $<0,0001$ \\
\hline Pernil (\%) & $34,57 \mathrm{a}$ & 34,62 a & $29,42 \mathrm{~b}$ & $30,71 \mathrm{ab}$ & $30,32 \mathrm{~b}$ & 6,56 & 31,93 & 0,001 \\
\hline Paleta (kg) & $0,84 \mathrm{~b}$ & $0,52 \mathrm{~b}$ & $1,87 \mathrm{a}$ & $1,77 \mathrm{a}$ & $1,74 \mathrm{a}$ & 18,94 & 1,35 & $<0,0001$ \\
\hline Paleta $(\%)$ & $19,28 \mathrm{~b}$ & $21,19 \mathrm{a}$ & $17,74 \mathrm{~b}$ & $19,47 \mathrm{ab}$ & $19,04 \mathrm{~b}$ & 5.04 & 19,34 & 0,0005 \\
\hline Costilhar (kg) & $1,53 \mathrm{~b}$ & $0,70 \mathrm{~b}$ & $4,22 \mathrm{a}$ & $3,28 \mathrm{a}$ & $3,40 \mathrm{a}$ & 23,33 & 2,63 & $<0,0001$ \\
\hline Costilhar (\%) & $34,30 \mathrm{~b}$ & $28,34 \mathrm{c}$ & $39,84 \mathrm{a}$ & $35,36 \mathrm{~b}$ & $37,33 a b$ & 5,45 & 35,03 & $<0,0001$ \\
\hline Rendimento de músculo (paleta) (\%) & 63,12 & 62,64 & 60,95 & 63,71 & 61,74 & 8,41 & 62,43 & 0,924 \\
\hline Rendimento de osso (paleta) (\%) & 29,57 & 28,49 & 23,21 & 25,93 & 23,93 & 26,23 & 26,23 & 0,08 \\
\hline Rendimento de gordura (paleta) (\%) & $7,30 \mathrm{c}$ & $8,87 \mathrm{bc}$ & $15,83 \mathrm{a}$ & $10,35 \mathrm{bc}$ & $14,33 \mathrm{ab}$ & 27,42 & 11,34 & 0,0012 \\
\hline
\end{tabular}

$\overline{\mathrm{CV}}$ - Coeficiente de variação; $\mathrm{P}$ - Probabilidade; $\mathrm{a}, \mathrm{b}, \mathrm{c}, \mathrm{d}, \mathrm{e}$ - Médias na linha seguidas por letras diferem $(\mathrm{P}<0,05)$ entre si.

No marmoreio foram observados valores maiores para os machos castrados e ovelhas, seguidos pelos machos inteiro e valores mínimos (traços de marmoreio) para borregas e cordeiros. 
As borregas apresentaram carne mais clara, seguida pelos animais adultos que foram semelhantes aos cordeiros (Tabela 5). Esperava-se que a carne de animais adultos apresentasse menor valor de luminosidade, uma carne mais escura e com maior valor de $a^{*}$, carne mais vermelha, devido ao acúmulo de mioglobina no músculo que ocorre com o passar da idade (Pinheiro et al., 2009).

Tabela 5. Médias observadas para características da carne de ovinos de diferentes categorias animais.

\begin{tabular}{|c|c|c|c|c|c|c|c|c|}
\hline \multirow{2}{*}{ Variáveis } & \multicolumn{5}{|c|}{ Categorias } & \multirow{2}{*}{$\begin{array}{l}\mathrm{CV} \\
(\%)\end{array}$} & \multirow{2}{*}{$\begin{array}{l}\text { Média } \\
\text { geral }\end{array}$} & \multirow{2}{*}{$\mathrm{P}$} \\
\hline & Borrega & Cordeiro & Macho castrado & Macho não castrado & Ovelha & & & \\
\hline$\overline{\mathrm{PH}}$ & 5,78 & 5,56 & 5,67 & 5,72 & 5,64 & 3,33 & 5,68 & 0,4176 \\
\hline Marmoreio & $1,20 \mathrm{~b}$ & $1,00 \mathrm{~b}$ & $4,40 \mathrm{a}$ & $2,40 \mathrm{ab}$ & $3,80 \mathrm{a}$ & 52,41 & 2,56 & 0,0017 \\
\hline L (luminosidade) & $46,69 \mathrm{a}$ & $36,22 \mathrm{~b}$ & $43,29 \mathrm{ab}$ & $43,09 \mathrm{ab}$ & $40,28 \mathrm{ab}$ & 9,6 & 41,91 & 0,0076 \\
\hline a (pigmento vermelho-azul) & $9,57 \mathrm{~b}$ & 16,87 a & $9,13 \mathrm{~b}$ & $13,39 \mathrm{ab}$ & $16,20 \mathrm{a}$ & 17,39 & 13,03 & $<0,0001$ \\
\hline $\mathrm{b}$ (pigmento amarelo - verde) & $13,85 \mathrm{a}$ & $8,54 \mathrm{c}$ & $12,33 \mathrm{ab}$ & $11,53 \mathrm{ab}$ & 9,86 bc & 13,33 & 11,22 & 0,0002 \\
\hline Croma & $16,85 \mathrm{ab}$ & $18,97 \mathrm{a}$ & $15,36 \mathrm{~b}$ & $17,90 \mathrm{ab}$ & 19,09 a & 10,12 & 17,63 & 0,0182 \\
\hline Tonalidade $\left(^{\circ}\right)$ & $55,34 \mathrm{a}$ & $26,81 \mathrm{~d}$ & $53,57 \mathrm{ab}$ & $41,18 \mathrm{bc}$ & $32,15 \mathrm{~cd}$ & 15,94 & 41,81 & $<0,0001$ \\
\hline Perda no descongelamento $(\%)$ & 11,71 & 7,94 & 9,75 & 6,95 & 10,81 & 31,7 & 9,43 & 0,109 \\
\hline Perda na cocção (\%) & 16,59 & 12,63 & 17,4 & 15,14 & 17,26 & 20,37 & 15,8 & 0,15 \\
\hline Força de cisalhamento $(\mathrm{KgF})$ & 4,95 & 5,5 & 4,93 & 5,38 & 6,52 & 26,14 & 5,46 & 0,415 \\
\hline
\end{tabular}

$\overline{\mathrm{CV}}$ - Coeficiente de variação; $\mathrm{P}$ - Probabilidade; a, b, c, d, e - Médias na linha seguidas por letras diferem $(\mathrm{P}<0,05)$ entre si.

$\mathrm{O}$ pigmento vermelho apresentou outro comportamento, carne mais vermelha foi observada para os cordeiros e ovelhas, seguidas pelos machos inteiros que foram semelhantes aos machos castrados e borregas. $\mathrm{O}$ pigmento amarelo, as borregas apresentaram maior valor, seguidas pelos machos, ovelhas e depois cordeiros. O croma seguiu comportamento semelhante ao pigmento $\mathrm{a}^{*}$ e a tonalidade semelhante ao pigmento $\mathrm{b}^{*}$.

Não foi observada diferença (Tabela 5) para a perda no descongelamento, cocção e força de cisalhamento $\mathrm{P}>0,05)$, e as médias observadas foram $9,43 \%, \quad 15,80 \%$ e $5,46 \mathrm{KgF}$, respectivamente. Interessante ressaltar que em valores absolutos a carne dos cordeiros não foi a mais macia.

A quantidade de cinzas e proteína não foi diferente entre as categorias animais $(\mathrm{P}>0,05)$ (Tabela 6). Entretanto a umidade e lipídios sofreram interferência da categoria animal, machos castrados e ovelhas apresentaram menor umidade e também apresentaram maior quantidade de lipídios.

Tabela 6. Médias observadas para análise centesimal da carne de ovinos de diferentes categorias animais.

\begin{tabular}{|c|c|c|c|c|c|c|c|c|}
\hline \multirow{2}{*}{ Variáveis } & \multicolumn{5}{|c|}{ Categorias } & \multirow{2}{*}{$\mathrm{CV}(\%)$} & \multirow{2}{*}{$\begin{array}{l}\text { Média } \\
\text { geral }\end{array}$} & \multirow{2}{*}{ Probabilidade } \\
\hline & Borrega & Cordeiro & Macho castrado & Macho não castrado & Ovelha & & & \\
\hline Umidade & $76,65 \mathrm{a}$ & $79,27 \mathrm{a}$ & $70,59 \mathrm{~b}$ & $75,81 \mathrm{a}$ & $71,65 \mathrm{~b}$ & 2,58 & 74,79 & $\overline{<0,0001}$ \\
\hline Cinzas & 1,03 & 1,01 & 0,99 & 1,03 & 1 & 6,22 & 1,01 & 0,848 \\
\hline Lipídios & $2,13 \mathrm{~b}$ & $1,06 \mathrm{~b}$ & $7,29 \mathrm{a}$ & $2,23 \mathrm{~b}$ & $6,07 \mathrm{a}$ & 42,7 & 13,93 & $<0,0001$ \\
\hline Proteína & 21,3 & 20,02 & 20,39 & 20,21 & 19,61 & 4,53 & 20,3 & 0,0911 \\
\hline
\end{tabular}

CV - Coeficiente de variação; a, b, c, d, e - Médias na linha seguidas por letras diferem $(\mathrm{P}<0,05)$ entre si.

Não houve diferença $(\mathrm{P}>0,05)$ para a intensidade de odor. As carnes foram classificadas com odor moderado. Quanto a maciez, as amostras foram classificadas como carne medianas. Na aceitabilidade, como ligeiramente aceitável. Apenas para suculência as amostras diferiram, onde as carnes de machos castrados, inteiros e ovelhas foram avaliadas como suculência moderada e as de borregas e cordeiros como pouca suculência. Resultados que reforçam a diferença observada no marmoreio, e mostram a percepção dos provadores mesmo em pequenas alterações. 
Tabela 7. Médias observadas para análise sensorial da carne de ovinos de diferentes categorias animais.

\begin{tabular}{lccccccc}
\hline \multirow{2}{*}{ Variáveis } & \multicolumn{3}{c}{ Categorias } & \multicolumn{3}{c}{ CV (\%) Probabilidade } \\
\cline { 2 - 7 } & Borrega & Cordeiro & Macho castrado & Macho não castrado & Ovelha & 0,1474 \\
\hline Intensidade de odor & 3,50 & 2,75 & 3,25 & 3,37 & 3,87 & 25,60 & 0,4603 \\
Maciez & 4,75 & 3,87 & 5,00 & 5,37 & 4,87 & 34,16 & 0,0336 \\
Suculência & $3,37 \mathrm{~b}$ & $3,37 \mathrm{~b}$ & $4,25 \mathrm{a}$ & $3,75 \mathrm{a}$ & $4,25 \mathrm{a}$ & 19,00 & 0,1204 \\
Aceitabilidade global & 6,50 & 4,62 & 5,87 & 6,00 & 6,87 & 28,79 & 0
\end{tabular}

$\overline{\mathrm{CV}}$ - Coeficiente de variação; a, b, c, d, e - Médias na linha seguidas por letras diferem $(\mathrm{P}<0,05)$ entre si.

\section{Conclusões}

Os machos castrados, machos inteiros e ovelhas apresentaram resultados com valores maiores que os cordeiros e borregas, isto levando em conta desempenho, tamanho de carcaça e cortes. Porém vale a pena ressaltar que carcaças menores das borregas e cordeiros apresentaram resultados satisfatórios. Deve se considerar o que o mercado consumidor almeja: consumir cortes grandes ou pequenos.

\section{Referências Bibliográficas}

ABNT. 1998. Associação Brasileira de Normas Técnicas - NBR 14141. Associação Brasileira de Normas Técnicas, Rio de Janeiro, RJ, BR.

AMSA. 1995. Research guidelines for cookery, sensory evaluation and instrumental tenderness measurements of fresh meat. In: Association, A. M. S. (ed.) American Meat Science Association. National Livestock and Meat Board, Savoy, IL.

AMSA - Americam Meat Science Association. 2001. Handbook Meat Evaluation. American Meat Science Association: Chicago.

ANUALPEC. 2017. Anuário da Pecuária Brasileira, 20th edn. Instituto FNP, São Paulo, SP, Brasil.

AOAC. 2005. - Association Official Analytical Chemist (2005), Official Methods of Analysis (18th ed.) edn. AOAC, Gaitherburg, Maryland, USA.

Beermann, D. H., Robinson, T. F. \& Hogue, D. E. 1995. Impact of composition manipulation on lean lamb production in the United States. Journal of Animal Science, 73, 2493-2502.

Beraldo, P.P. 2014. Consumo de carne ovina: desafios de produção e abate clandestino. Disponível

$\mathrm{em}$ :

$<$ http://ruralcentro.uol.com.br/noticias/con sumo-de-carne-ovina-desafios-deproducao-e-abate-clandestino75435\#y=201 >. [Acesso em: 15 jul 2014].
Beserra, F.J., Nassu, R.T., Melo, L.R.R., Rodrigues, M. C. P. \& Silva, E. M. C. 1999. Manufacturing of a restructured hamlike product with goat meat. In: IFT Annual Meeting, Chicago, 1999. Book of abstracts, Chicago: IFT, p.89.

Cañeque, V. \& Sañudo, C. 2000. Metodologia para el Estúdio de la Calidad de la Canal $y$ de la Carne em Ruminantes. INIA: Madrid.

Cezar, M. \& Sousa, W. 2007. Carcaças ovinas e caprinas: obtenção, avaliação e classificação. Uberaba: Editora Agropecuária Tropical, 147.

CIE. 1986. Colorimetry. Commission Internationale de l'Eclairage. 2 ed. Commission Internationale de l'Eclairage, Vienna.

Gonçalves, L. A. G., Zapata, J. F. F., Rodrigues, M. C. P. \& Borges, A. S. 2004. Efeitos do sexo e do tempo de maturação sobre a qualidade da carne ovina. Ciência e Tecnologia de Alimentos, 24, 459-467.

Lawrie, R. 1981. Developments in meat science-2. Applied Science Publishers.

Osório, J. C. S. \& Osório, M. T. 2005. Produção de carne ovina: técnicas de avaliação "in vivo" e na carcaça. Universidade Federal de Pelotas, Pelotas.

Pearce, K. L., Rosenvold, K., Andersen, H. J. \& Hopkins, D. L. 2011. Water distribution and mobility in meat during the conversion of muscle to meat and ageing and the impacts on fresh meat quality attributes - A review. Meat Science, 89, 111-124.

Pinheiro, R. S. B., Jorge, A. M., Souza, H. B. A. \& Boiago, M. M. 2009. Coloração da gordura e qualidade da carne de ovelhas de descarte abatidas em distintos estágios fisiológicos. Arquivo Brasileiro de Medicina Veterinária e Zootecnia, 62, 468-474. 
Pinheiro, R. S. B., Silva Sobrinho, A. G., Souza, H. B. A. \& Yamamoto, S. M. 2009. Qualidade de carnes provenientes de cortes da carcaça de cordeiros e de ovinos adultos. Revista Brasileira de Zootecnia, 38, 1790-1796.

Ramos, E.D. \& Gomide, L.A.M. 2007. Avaliação $d a$ qualidade de carnes: fundamentos $\mathrm{e}$ metodologias. Ed.UFV: Viçosa.

SAS. 2004. SAS/STAT User guide, Version 9.1.2. SAS Institute Inc, Cary, NC, USA.

SEBRAE - Serviço brasileiro de apoio às micro e pequenas empresas. 2005. Informações de mercado sobre caprinos e ovinos. Brasil. Disponível em: $<$ http://www.bibliotecas.sebrae.com.br/chronu s/ARQUIVOS CHRONUS/bds/bds.nsf/40B6 5B09464CA07D032571540041EC16/\$File/N T0003202E.pdf>. [Acesso em: 17 Set 2017]

Siqueira, E. R. \& Fernandes, S. 1999. Pesos, rendimentos e perdas da carcaça de cordeiros Corriedale e mestiços Ile de France $\mathrm{x}$ Corriedale, terminados em confinamento. Ciência Rural, 29, 143-148.
Wheeler, T. L., Shackelford, S. D. \& Koohmaraie, M. 1997. Standardizing collection and interpretation of Warner-Bratzler shear force and sensory tenderness data. Proceding Reciprocal Meat Conference. Citeseer, USA.

Yáñez, E. A., Resende, K. T., Ferreira, A., Medeiros, A. N., Silva Sobrinho, A. G., Pereira Filho, J. M., Teixeira, I. A. M. A. \& Artoni, S. M. B. 2004. Utilização de medidas biométricas para predizer características da carcaça de cabritos Saanen. Revista Brasileira de Zootecnia, 33, 1564-1572.

Zeola, N. M. B., Souza, P. A., Souza, H. B. A. \& Silva Sobrinho, A. G. 2007. Parâmetros qualitativos da carne ovina: um enfoque à maturação e marinação. Revista Portuguesa de Ciências Veterinárias, 102, 215-224.

Article History:

Received 16 October 2017

Accepted 11 November 2017

Available online 20 January 2018

License information: This is an open-access article distributed under the terms of the Creative Commons Attribution License 4.0, which permits unrestricted use, distribution, and reproduction in any medium, provided the original work is properly cited. 